\title{
On the Modified Tikhonov's Regularization Method for the Cauchy Problem of the Laplace Equation
}

\author{
Jiang-Ren Chang \\ Department of System Engineering \& Naval Architecture \\ Weichung Yeih \\ Department of Harbor and River Engineering, National Taiwan Ocean University Keelung, Taiwan 202, ROC. \\ Min-Harng Shieh \\ Department of Harbor and River Engineering, National Taiwan Ocean University Keelung, Taiwan 202, ROC.
}

Follow this and additional works at: https://jmstt.ntou.edu.tw/journal

Part of the Engineering Commons

\section{Recommended Citation}

Chang, Jiang-Ren; Yeih, Weichung; and Shieh, Min-Harng (2001) "On the Modified Tikhonov's Regularization Method for the Cauchy Problem of the Laplace Equation," Journal of Marine Science and Technology: Vol. 9: Iss. 2, Article 6. DOI: 10.51400/2709-6998.2441

Available at: https://jmstt.ntou.edu.tw/journal/vol9/iss2/6

This Research Article is brought to you for free and open access by Journal of Marine Science and Technology. It has been accepted for inclusion in Journal of Marine Science and Technology by an authorized editor of Journal of Marine Science and Technology. 


\title{
ON THE MODIFIED TIKHONOV'S REGULARIZATION METHOD FOR THE CAUCHY PROBLEM OF THE LAPLACE EQUATION
}

\author{
Jiang-Ren Chang*, Weichung Yeih**, Min-Harng Shieh**
}

\author{
Key words: Tikhonov's regularization method, singular value deco- \\ mposition, L-curve, modified Tikhonov's regularization \\ method, Cauchy problem.
}

\begin{abstract}
In this paper, an inverse problem of the Laplace equation with Cauchy data is examined. Due to the ill-posed behavior of this inverse problem, the Tikhonov's regularization technique is employed and the L-curve concept is adopted to determine the optimal regularization parameter. Also, the singular value decomposition method is used in conjunction with the L-curve concept for the same problem. Numerical results show that neither the traditional Tikhonov's regularization method nor the singular value decomposition method can yield acceptable results when the influence matrix is highly ill-posed. A modified regularization method, which combines the singular value decomposition method and regularization method, is thus proposed, and this new method shows that it is a better way to treat this kind of inverse problems comparing with the other two traditional methods. Numerical results also show that the inverse problem with Cauchy data is better to formulate by the singular integral equation than by the hypersingular integral equation for the constant element scheme. The inverted boundary data becomes closer to the exact solution when the number of elements increases, and numerical experiments show that the rate of convergence is higher for the formulation using the singular integral equation. Numerical experiments are made to examine how the boundary Cauchy data affect the inverted process. It is concluded that the inversion of unknown boundary data is more effective when the Cauchy data are given more precisely and are distributed on the whole boundary more diversely.
\end{abstract}

\section{INTRODUCTION}

The inverse problem is a complementary part of the direct problem in the mathematical language since it is defined as a problem that does not belong to the direct problem [1]. The inverse problem is more difficult to work out due to its ill-posed behavior. Hadamard [2] defined that a well-posed problem must satisfy three requirements: (1) existence of solution; (2) uniqueness of solution and (3) continuous dependence of solution

Paper Received August 1, 2000. Author for Correspondence: Jiang-Ren Chang. *Department of System Engineering \& Naval Architecture

**Department of Harbor and River Engineering, National Taiwan Ocean University Keelung, Taiwan 202, ROC. on data, and he said a problem which does not satisfy any one of these three requirements is ill-posed. Being compared to the direct problem, the inverse problem is unfortunately ill-posed in most cases. To the authors' knowledge, most inverse problems do not have the existence theorem so far. And the existence of solution is usually assumed to be true in most inverse problems. Mathematically speaking, the existence theorem of solution certainly is important. However, the existence of solution is sometimes not very meaningful in practical applications since data usually contain error, which might result in no solution at all [3]. The uniqueness of solution is proved to be true for some cases $[4,5]$. However, the uniqueness of solution is not true in some cases. For example, Gau and Mura [6] proved that it is impossible to invert the residual plastic strain in a domain by the residual boundary displacements. The reason for the nonuniqueness of solution is that the information given is not enough since the plastic strain inside the plastic zone depends on the loading history and the same residual boundary displacement data can be obtained by different loading paths. Even when the existence and uniqueness of solution is guaranteed, inverse problems are not easy to be solved due to the very sensitive nature of solution on the given data. It means a little perturbation on the data may result in a large amount of error in the solution.

The Cauchy problem is defined as the complete boundary data that are given on part of the boundary and no boundary data are prescribed on the remaining part. Yeih et al. [3] solved the Cauchy problem for an elstostatic field and further formulated this problem by the fictitious boundary element method [7] and the Tikhonov regularization method. The Cauchy problem in the Laplace equation for an infinite strip has been examined [8].

To treat the ill-posed behavior of inverse problems, several techniques are available. Tikhonov [9] proposed a regularization method that is derived from a minimization problem with a constraint condition. Another well-known approach is the singular value decomposition method (SVD) [10], which produces a 
pseudo-inverse matrix by omitting the unreasonable contribution from small singular values below a threshold value.

To select the optimal regularization parameter in the Tikhonov's regularization method or the threshold value in the SVD, two approaches are frequently used. The first method is to set the threshold value of the objective functional artificially, then the best regularization parameter in the regularization method and the best threshold value in the singular value decomposition method can be determined by an iteration process, in which the first value of these parameters to make the objective functional be less than the threshold value is chosen to be the best parameter. However, the threshold value of the objective functional is sometimes not so easy to be determined and is mainly based on the experience. Another approach is the so-called L-curve concept [11]. Basically, this method can search for all possible parameters and calculate two indices, one represents the sensitivity of the solution and the other one represents the distorted behavior of the system. The best parameter is selected by finding the corner of the Lcurve, and this best parameter does not make solution too sensitive and distort the system too much at the same time theoretically. The details of L-curve concept will be explained in the following section.

In this paper, the Cauchy problem for the Laplace equation is reexamined. The Tikhonov's regularization method and singular value decomposition method are employed with the L-curve concept. It is found that both methods cannot construct the unknown boundary data effectively when the ill-posed behavior of the system is extremely serious. A new method that combines the concept of the Tikhonov's regularization method with the singular value decomposition method is proposed. Numerical experiments show that the proposed method yields better results than the other two traditional methods. Several numerical examples are also included to show how the information of data influences the solution.

\section{PROBLEM DEFINITION AND FORMULATIONS CONSTRUCTED BY SINGULAR AND HYPERSINGULAR INTEGRAL EQUATIONS}

The Cauchy problem is defined as follows. The governing equation that the physical quantities satisfy inside the domain is the Laplace equation. For an arbitrary domain $D$ enclosed by the boundary $B$, as shown in Fig. 1, the boundary can be separated into two parts: one is $B_{k}$ where the potential and the normal derivative of the potential are given at the same time, and the other is $B_{u}$ where both the potential and the normal derivative of the potential are unknowns. The

$$
\mathrm{B}_{\mathrm{k}}: \mathrm{u}, \mathrm{t} \text { known }
$$

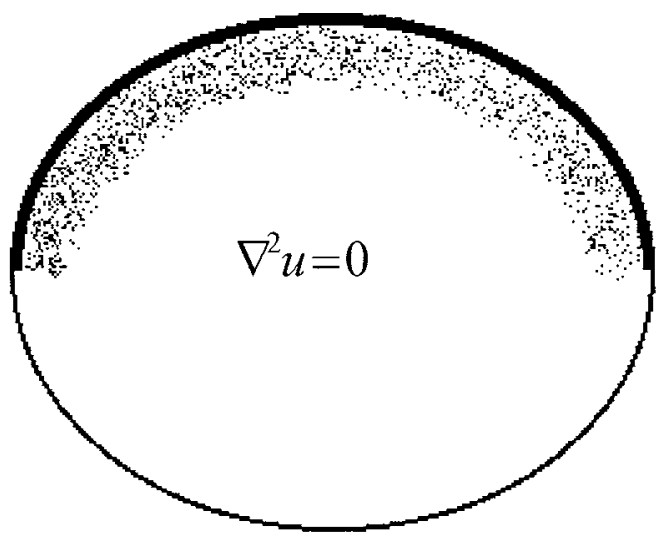

$\mathrm{B}_{\mathrm{u}}: \mathrm{u}, \mathrm{t}$ unknown

Fig. 1. Illustrative diagram of problem setup.

inverse problem is stated as follows. For the given boundary data on $B_{k}$, can one invert the unknown boundary data on $B_{u}$ such that one can reconstruct the solution of every point inside the domain $D$ ?

To formulate this problem by the boundary integral equations, the following two equations are constructed for $x$ point on the smooth boundary:

$$
\begin{aligned}
\pi u(x) & =\text { C.P.V. } \int_{B} T(s, x) u(s) d B(s) \\
& -R . P . V \cdot \int_{B} U(s, x) t(s) d B(s) \\
\pi t(x) & =H . P . V \cdot \int_{B} M(s, x) u(s) d B(s) \\
& - \text { C.P.V. } \int_{B} L(s, x) t(s) d B(s)
\end{aligned}
$$

where $u(x)$ is the single layer potential on the boundary, $t(x) \equiv \frac{\partial u(x)}{\partial n_{x}}$ is the normal derivative of the $u(x)$ with $n_{x}$ representing the outnormal direction at $x$ point, $U(s, x)$ is the fundamental solution which satisfies $\nabla_{x}^{2} U(s, x)=$ $2 \pi \delta(x-s)$ and is expressed as $\ln |x-s|, T(s, x) \equiv \frac{\partial u(s, x)}{\partial n_{s}}$, $L(s, x) \equiv \frac{\partial u(s, x)}{\partial n_{s}}$ and $M(s, x) \equiv \frac{\partial^{2} U(s, x)}{\partial n_{s} \partial n_{x}}$. In the above two equations, C.P.V., R.P.V. and H.P.V. represent Cauchy principal value, Riemann principal value and Hadamard principal value, respectively. Eqs. (1) and (2) together are named as the dual boundary element method by Chen and Hong [12]. Eq. (1) is also called 
the singular integral equation (UT equation) and Eq. (2) is called the hypersingular integral equation (LM equation) by the singular nature of the kernels.

To study the Cauchy problem, Eqs. (1) and (2) can be rearranged into the following discretized forms by gathering the unknown quantities and known information on the opposite sides of the equations.

For the collocation point $x_{i}$ of the $i$-th element is on $B_{k}$, we have

$$
\begin{aligned}
&- \sum_{\substack{j=1 \\
\left(B_{j} \in B_{u}\right)}}^{n} u\left(s_{j}\right) \int_{B_{j}} T\left(s_{j}, x_{i}\right) d B\left(s_{j}\right) \\
&+ \sum_{\substack{j=1 \\
\left(B_{j} \in B_{u}\right)}}^{n} t\left(s_{j}\right) \int_{B_{j}} U\left(s_{j}, x_{i}\right) d B\left(s_{j}\right) \\
&= \sum_{\substack{j=1 \\
\left(B_{j} \in B_{k}\right)}}^{n} u\left(s_{j}\right) \int_{B_{j}} T\left(s_{j}, x_{i}\right) d B\left(s_{j}\right)-\pi u\left(x_{i}\right) \\
&- \sum_{\substack{j=1 \\
\left(B_{j} \in B_{k}\right)}}^{n} t\left(s_{j}\right) \int_{B_{j}} U\left(s_{j}, x_{i}\right) d B\left(s_{j}\right), \\
& \sum_{\substack{j=1 \\
\left(B_{j} \in B_{u}\right)}}^{n} u\left(s_{j}\right) \int_{B_{j}} M\left(s_{j}, x_{i}\right) d B\left(s_{j}\right) \\
&+ \sum_{\substack{j=1 \\
\left(B_{j} \in B_{u}\right)}}^{n} t\left(s_{j}\right) \int_{B_{j}} L\left(s_{j}, x_{i}\right) d B\left(s_{j}\right) \\
&= \sum_{\substack{j=1 \\
\left(B_{j} \in B_{k}\right)}}^{n} u\left(s_{j}\right) \int_{B_{j}} M\left(s_{j}, x_{i}\right) d B\left(s_{j}\right)-\pi u\left(x_{i}\right) \\
&-\sum_{\substack{j=1 \\
\left(B_{j} \in B_{k}\right)}}^{n} t\left(s_{j}\right) \iint_{B_{j}} L\left(s_{j}, x_{i}\right) d B\left(s_{j}\right),
\end{aligned}
$$

For the collocation point $x_{i}$ of the $i$-th element is on $B_{u}$, we have

$$
\begin{aligned}
& \pi u\left(x_{i}\right)-\sum_{\substack{j=1 \\
\left(B_{j} \in B_{u}\right)}}^{n} u\left(s_{j}\right) \int_{B_{j}} T\left(s_{j}, x_{i}\right) d B\left(s_{j}\right) \\
&+\sum_{\substack{j=1 \\
\left(B_{j} \in B_{u}\right)}}^{n} t\left(s_{j}\right) \int_{B_{j}} U\left(s_{j}, x_{i}\right) d B\left(s_{j}\right) \\
&=\sum_{\substack{j=1 \\
\left(B_{j} \in B_{k}\right)}}^{n} u\left(s_{j}\right) \int_{B_{j}} T\left(s_{j}, x_{i}\right) d B\left(s_{j}\right) \\
&-\sum_{\substack{j=1 \\
\left(B_{j} \in B_{k}\right)}}^{n} t\left(s_{j}\right) \int_{B_{j}} U\left(s_{j}, x_{i}\right) d B\left(s_{j}\right), \\
& \sum_{\substack{j=1 \\
\left(B_{j} \in B_{u}\right)}}^{n} u\left(s_{j}\right) \int_{B_{j}} M\left(s_{j}, x_{i}\right) d B\left(s_{j}\right)+\pi t\left(x_{i}\right) \\
&+\sum_{\substack{j=1 \\
\left(B_{j} \in B_{u}\right)}}^{n} t\left(s_{j}\right) \int_{B_{j}} L\left(s_{j}, x_{i}\right) d B\left(s_{j}\right)
\end{aligned}
$$

$$
\begin{aligned}
& =\sum_{\substack{j=1 \\
\left(B_{j} \in B_{k}\right)}}^{n} u\left(s_{j}\right) \int_{B_{j}} M\left(s_{j}, x_{i}\right) d B\left(s_{j}\right) \\
& -\sum_{\substack{j=1 \\
\left(B_{j} \in B_{k}\right)}}^{n} t\left(s_{j}\right) \int_{B_{j}} L\left(s_{j}, x_{i}\right) d B\left(s_{j}\right) .
\end{aligned}
$$

Rearranging Eqs. (3a) and (4a) together (or 3(b) and 4(b) together) into a matrix form, we obtain the linear equations for singular and hypersingular formulations, respectively. And the linear equations can be generally written as

$$
\mathbf{A x}=\mathbf{b}
$$

where $\mathbf{A}$ is the influence matrix, $\mathbf{x}$ is the unknown vector and $\mathbf{b}$ is the information vector. It is noted that the dimension of the leading matrix $\mathbf{A}$ is not necessary a square matrix since the element numbers on $B_{k}$ and $B_{u}$ are not necessary the same.

\section{TIKHONOV'S REGULARIZATION METHOD, SINGULAR VALUE DECOMPOSITION, L-CURVE AND MODIFIED TIKHONOV'S REGULARIZATION METHOD}

For the Cauchy problem of the Laplace equation, the influence matrix $\mathbf{A}$ is often ill-posed such that the regularization technique which regularizes the influence matrix is necessary. Tikhonov [9] proposed a method to transform this ill-posed problem into a wellposed one. Instead of solving Eq. (5) directly, he proposed to solve the following problem:

$$
\text { minimize }\|\mathbf{x}\|^{2} \text { subject to }\|\mathbf{A} \mathbf{x}-\mathbf{b}\|^{2} \leq \varepsilon
$$

where $\varepsilon$ is the prescribed error tolerance. The proposed problem in Eq. (6) is equivalent to: [6]

$$
\text { minimize }\|\mathbf{A} \mathbf{x}-\mathbf{b}\|^{2} \text { subject to }\|\mathbf{x}\|^{2} \leq \varepsilon^{*},
$$

and the Euler-Lagrange equation obtained from Eq. (7) is written as

$$
\left(\mathbf{A}^{T} \mathbf{A}+\alpha I\right) \mathbf{x}=\mathbf{A}^{T} \mathbf{b}
$$

where $\alpha$ is the regularization parameter (Lagrange parameter).

To solve Eq. (8), the nonlinear iteration method should be used since the two unknown quantities, $\alpha$ and $\mathbf{x}$, are involved. To determine the regularization method, an iteration method based on a prescribed error range, $\varepsilon$, has been proposed [6,7]. However, determination of the prescribed error range depends on the engineering judgment. 
Another method is the so-called L-curve concept, which will be elaborated on later. Another approach to solve this ill-posed problem is the singular value decomposition method. The singular value decomposition method decomposes the influence matrix $\mathbf{A}$ into the following form:

$$
\mathbf{A}=\mathbf{U} \Sigma \mathbf{V}^{T}
$$

where $\mathbf{U}$ and $\mathbf{V}$ are unitary matrices constructed by the left and right singular vectors, and $\Sigma=\operatorname{diag}\left\{\sigma_{1}, \sigma_{2}, \ldots\right.$, $\left.\sigma_{n-1}, \sigma_{n}\right\}$ where $\sigma_{1}, \sigma_{2}, \ldots, \sigma_{n-1}, \sigma_{n}$ are singular values [10]. The psuedo-inverse matrix, $A^{+}$, then can be constructed by the following equation:

$$
\mathbf{A}^{+}=\mathbf{V} \Sigma^{-1} \mathbf{U}^{T} .
$$

When the problem is ill-posed, some singular values will tend to zero (or more precisely speaking, some singular values will become too small comparing with the largest singular value). Therefore, $\Sigma^{-1}$ will contain some quantities tend to infinite large which is for sure not practical in numerical implementation. For an illposed problem, $\Sigma^{-1}$ is replaced by $\Sigma^{+}$in which the diagonal elements are determined by the following rule:

(1) determine a prescribed threshold value, $\gamma$, and the maximum singular value $\sigma_{\max }$;

(2) if $\sigma_{i} \geq \gamma \bullet \sigma_{\max }$, the corresponding element in $\gamma$ is $\Sigma^{+}$ written as $\frac{1}{\sigma}$;

(3) if $\sigma_{i}<\gamma \cdot \sigma_{\max }$, the corresponding element in $\Sigma^{+}$is written as 0 .

The singular value decomposition method actually neglects the unreasonable contribution from small singular values since errors will be enlarged by these small singular values.

In the above two popular methods, they all require some prescribed values which is determined artificially. However, to select an optimal prescribed value is sometimes not so easy. The L-curve concept [11] is proposed to aid us in selecting the best parameter. Two indices are frequently used, one represents the sensitivity of the influence matrix on the solution and the other represents the degree of distortion to the original system. Usually, the square of the norm of the unknown vector, $\|\mathbf{x}\|^{2}$, is chosen as the index of sensitivity and $\|\mathbf{A x}-\mathbf{b}\|^{2}$ is chosen as the index of degree of distortion. In the logarithm scale, a typical diagram for the Tikhonov regularization method combined with the L-curve concept is illustrated in Fig. 2. One can find that when the regularization parameter, $\alpha$, is small, $\|\mathbf{x}\|^{2}$ tends to very large even though $\|\mathbf{A x}-\mathbf{b}\|^{2}$ is small. It is shown that the regularization parameter is too small such that not much improvement in the influence matrix is done. On the other hand, when the regularization parameter, $\alpha$, is large, $\|\mathbf{A x}-\mathbf{b}\|^{2}$ tends to be very large even though $\|\mathbf{x}\|^{2}$ tends to small value which shows that the regularization parameter is too large such that the original system is distorted too much. Therefore, the compromised results of $\|\mathbf{x}\|^{2}$ and $\|\mathbf{A x}-\mathbf{b}\|^{2}$ lead us to choose the corresponding value in the corner of the L-shape curve as the optimal regularization parameter. The same technique can be used for the singular value decomposition method for choosing the threshold value, $\gamma$. Although application of the L-curve concept in the singular value decomposition method has not been adopted to the authors' knowledge, this concept is adopted in this paper.

It is seen in Eq. (9) that the traditional Tikhonov's regularization method adds a parameter, $\alpha$, in the diagonal terms in the matrix $\mathbf{A}^{T} \mathbf{A}$. However, the generalized Tikhonov's regularization method suggested the square of any convergent norm $\|\mathbf{H x}\|^{2}$ can be adopted in Eq. (6) as the objective functional in stead of using $\|\mathbf{x}\|^{2}$, where $\mathbf{H}$ is the prescribed operator. Based on this concept, the modified Tikhonov's regularization method is proposed as follows:

$$
\text { minimize }\|\mathbf{H} \mathbf{x}\|^{2} \text { subject to }\|\mathbf{A x}-\mathbf{b}\|^{2} \leq \varepsilon
$$

where $\mathbf{H}$ is defined as

$$
\left[H_{i j}\right] \equiv\left\{\begin{array}{rr}
1 & \text { when } i=j \text { and } \sigma_{i} \geq \gamma \sigma_{\max } \\
0 & \text { all other elements }
\end{array} .\right.
$$

The corresponding Euler-Lagrange equation is found as

$\left(\mathbf{A}^{T} \mathbf{A}+\alpha \mathbf{H}\right) \mathbf{x}=\mathbf{A}^{T} \mathbf{b}$.

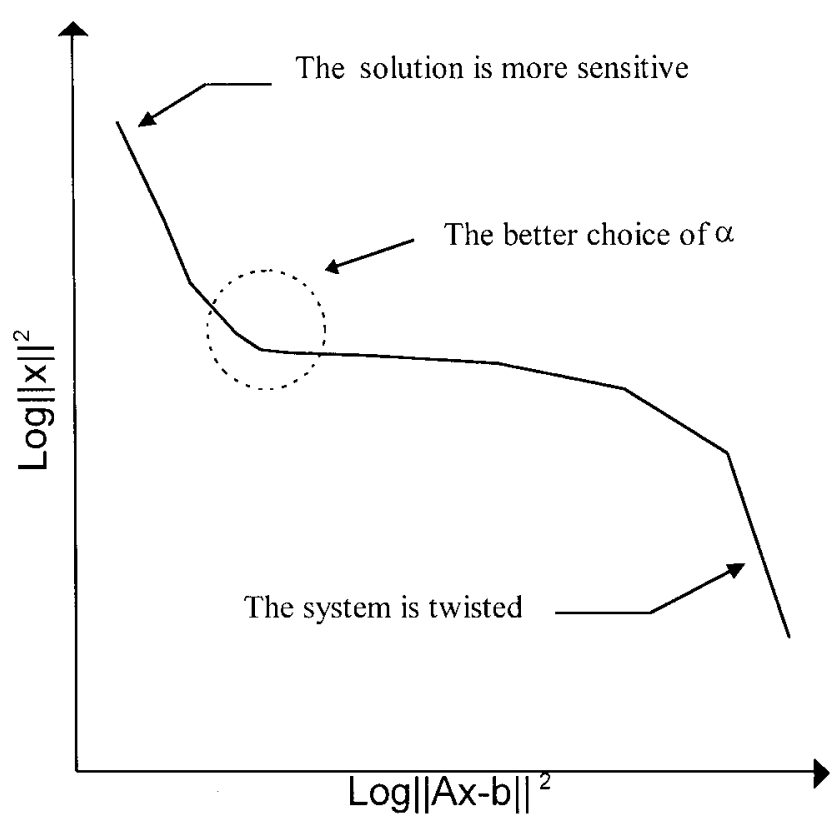

Fig. 2. The concept of the L-curve. 
Comparing Eq. (12) with Eq. (8), it is found that this modified regularization method only adds the regularization parameter in the diagonal elements whose corresponding singular values are above the required threshold value.

It is worth mentioned here that both the traditional regularization method and the singular value decomposition method have one 'regularization' parameter, however, there exist two parameters in the modified regularization method since it combines the spirits of these two methods. Furthermore, the number of indices representing the degree of distortion should become two in the modified regularization method; and these two indices are $\|\mathbf{A x}-\mathbf{b}\|^{2}$ and $\gamma$. As shown in Fig. 3, the shape of $\|\mathbf{x}\|^{2}$ versus curve is not the L-curve when $\| \mathbf{A x}$ $-\mathbf{b} \|^{2}$ is too small and the shape of $\|\mathbf{x}\|^{2}$ versus $\|\mathbf{A x}-\mathbf{b}\|^{2}$ curve becomes L-curve when $\gamma$ exceeds some value. Therefore, it is concluded the best parameters one should choose is the corresponding $\alpha$ value at the corner of the very first L-curve and in which the $\gamma$ value can be determined.

\section{NUMERICAL RESULTS AND DISCUSSION}

Two examples are given as shown in Fig. 4. For the circular disc with radius equal to 1 , the Cauchy boundary conditions are given as $u=r \sin \theta=\sin \theta$ and $t$ $=\frac{\partial u}{\partial n}=\sin \theta$ for $r=1$ and $0 \leq \theta \leq \pi$. In this circular disc example, several numerical experiments are implemented to check for: (1) the performance between SVD, Tikhonov's regularization method and modified regularization method; (2) the performance between the singular integral equation method and the hypersingular integral equation method; (3) the convergence of solutions; (4) the effect of information on the solution by equal length elements on the known and unknown boundaries; (5) the effect of information accuracy on the solution by equal element numbers on the known and unknown boundaries.

Another example is a square with the length of each side equal to 1 . The Cauchy boundary conditions are given on partial boundary such that the exact solution in the whole domain is $u(x, y)=x y$. In this example, we examine how the diversity of boundary data will affect the solution. For given Cauchy boundary conditions on half of the boundary, four kinds of distributions are given. For details of numerical examples, please refer to Table 1. In this table, the numerical examples are labeled with character $\mathrm{C}$ representing the circular case and $S$ representing the square one. Besides, the boundary with known Cauchy data for each case are listed according to Fig. 4, further, the number of elements on $B_{k}$ and $B_{u}$ and the optimal parameter $\gamma$ and $\alpha$ for each case are also listed, respectively.

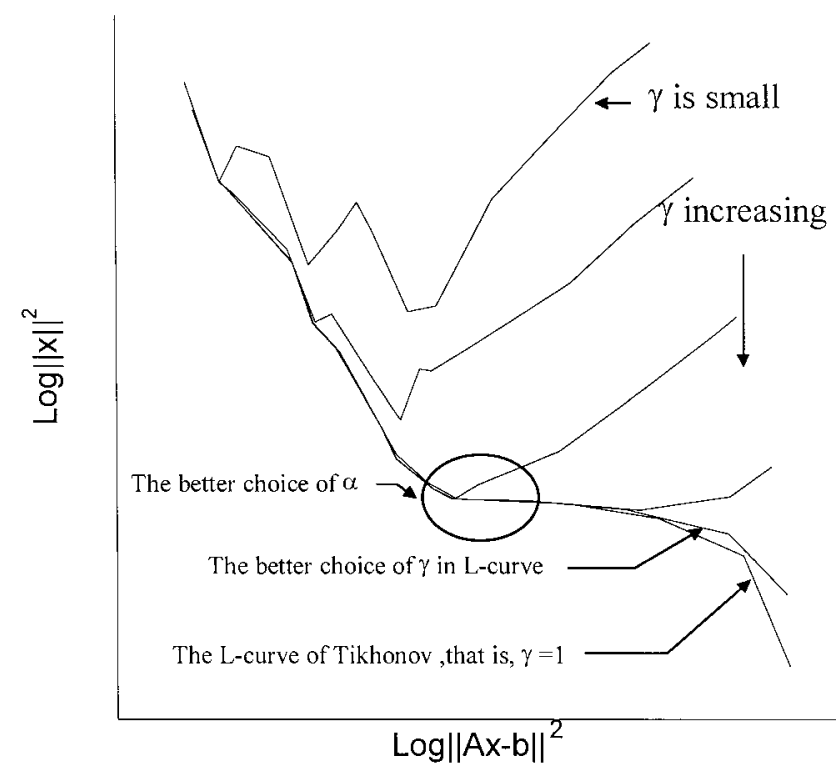

Fig. 3. Illustrative example for selecting parameters in the modified regularization method.
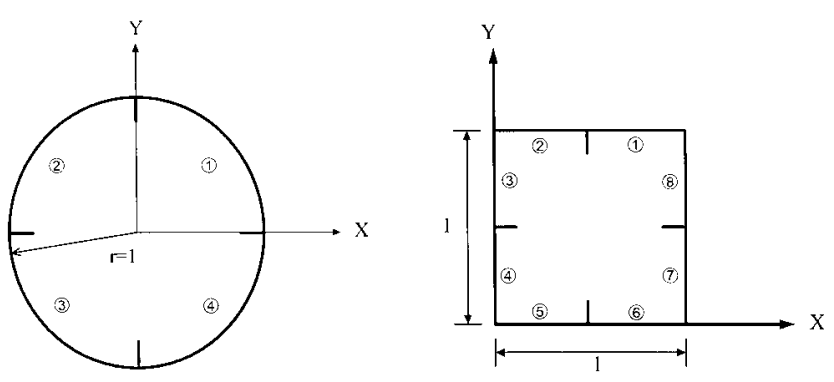

Fig. 4. Two numerical examples for the inverse problems.

Fig. 5 shows the results of boundary potential, $u$, on the unknown boundary by using the SVD method. It is clearly shown that the results do not converge to the exact solution when the number of elements increases. This result stems from the numerical inaccuracy of the computer and SVD algorithm. In SVD method, an iteration process should be used in order to perform the singular-value decomposition. However, the truncated error large when the dimensions of leading matrix becomes are large. In computation reality, the dimension of the matrix should be better less than a hundred [13]. A dimension of the matrix larger than 100 will cause computational difficulty for the personal computer used nowadays, our results with 144 elements just confirm this. It is concluded that although the SVD method can obtain a reasonable solution theoretically, it cannot obtain a better solution when the number of elements increases too much such that the truncate error becomes dominated. 
Table 1. Boundary conditions, elements and best parameters

\begin{tabular}{|c|c|c|c|c|c|c|c|}
\hline \multirow{2}{*}{ Label } & \multirow{2}{*}{$B_{k}$} & \multicolumn{2}{|c|}{ no. of elements } & \multicolumn{2}{|c|}{ UTequation } & \multicolumn{2}{|c|}{ LM equation } \\
\hline & & $B_{k}$ & $B_{u}$ & $\gamma$ & $\alpha$ & $\gamma$ & $\alpha$ \\
\hline $\mathrm{C} 1$ & (1)(2) & 36 & 36 & $5.813 \mathrm{E}-3$ & 1.E-8 & $2.733 \mathrm{E}-5$ & 1.E-3 \\
\hline $\mathrm{C} 2$ & (1) (2) & 72 & 72 & $5.028 \mathrm{E}-3$ & 1.E-8 & $3.593 \mathrm{E}-5$ & 1.E-3 \\
\hline $\mathrm{C} 3$ & (1)(2) & 144 & 144 & N.A & N.A & $5.396 \mathrm{E}-6$ & 1.E-3 \\
\hline $\mathrm{C} 4$ & (1) & 36 & 108 & $4.035 \mathrm{E}-3$ & 1.E-9 & $8.129 \mathrm{E}-6$ & 1.E-7 \\
\hline $\mathrm{C} 5$ & (1) (2) (3) & 108 & 36 & 7.98E-17 & 1.E-5 & $2.06 \mathrm{E}-17$ & 1.E-2 \\
\hline C6 & (1) & 72 & 72 & $5.146 \mathrm{E}-3$ & 1.E-9 & $1.414 \mathrm{E}-4$ & 1.E-6 \\
\hline $\mathrm{C} 7$ & (1) (2) (3) & 72 & 72 & $1.639 \mathrm{E}-3$ & 1.E-7 & $2.570 \mathrm{E}-5$ & 1.E-2 \\
\hline S1 & (1) (2) (3)(4) & 100 & 100 & $1.135 \mathrm{E}-3$ & 1.E-7 & $2.184 \mathrm{E}-5$ & 1.E-4 \\
\hline S2 & (1) (2) (5) (6) & 100 & 100 & $7.048 \mathrm{E}-4$ & 1.E-12 & $1.058 \mathrm{E}-5$ & 1.E-8 \\
\hline S3 & (1)(3) 5 (7) & 100 & 100 & $1.340 \mathrm{E}-3$ & 1.E-10 & $5.803 \mathrm{E}-6$ & 1.E-8 \\
\hline
\end{tabular}

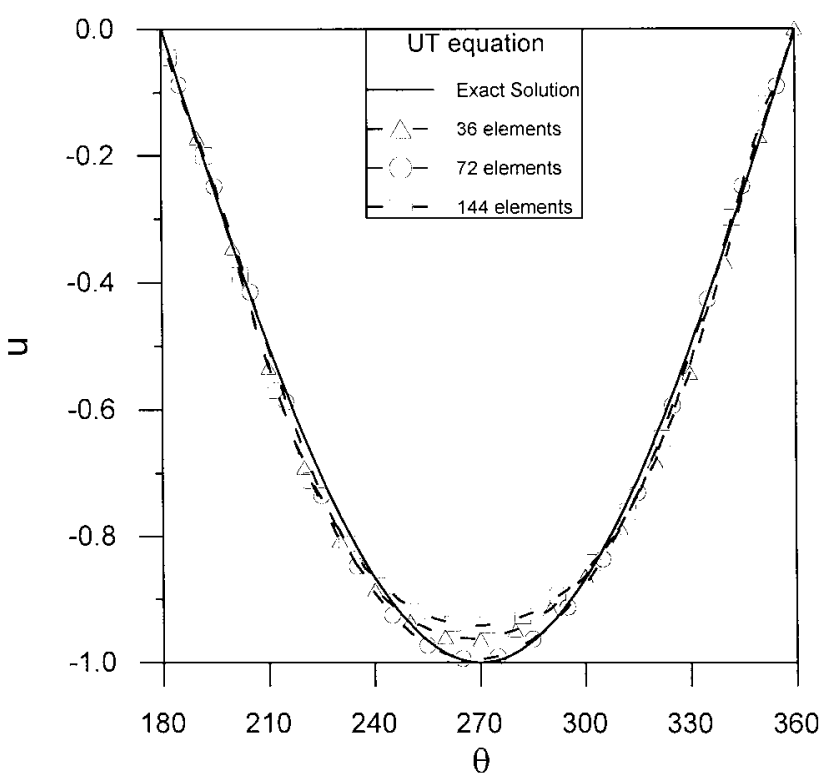

Fig. 5. Inverted potential on $B_{u}$ using SVD method.

Fig. 6 shows the boundary potential, $u$, on the unknown side for the circular disc case by using Tikhonov's regularization method and the modified regularization method (36 elements totally). It is seen that the modified regularization method performs better than the Tikhonov's regularization method. It is concluded that the modified regularization method is a better choice than the other two methods. The convergence of modified regularization method with respect to element number will be illustrated later. Figs. 7(a) and 7(b) illustrate the unknown potential, $u$, for the circular cases by using the singular integral equation method and the hypersingular integral equation, respectively. It is shown that the convergent speed of the singular integral equation is faster than that of the hypersingular

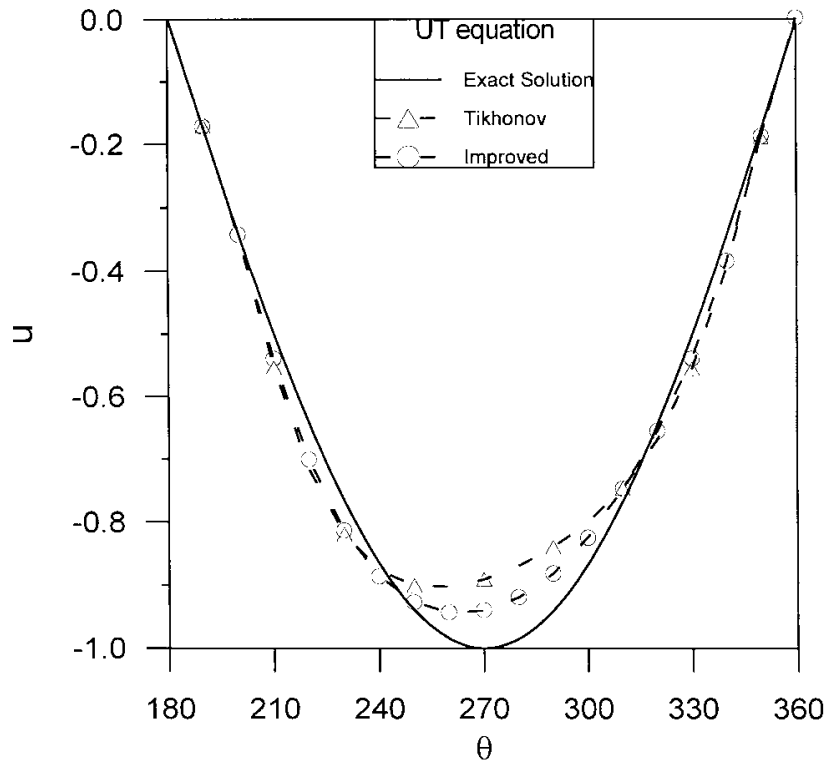

Fig. 6. Inverted potential on $B_{u}$ using Tikhonov's regularization method and modified method.

integral equation. Furthermore, the solution obtained from the singular integral equation is more close to the exact solution than that obtained from the hypersingular integral equation for the same element number discretization.

For the case of equal length element discretization on the circular disc case (example C4, C2 and C5), the Cauchy information is given on a quarter, a half and three quarters of the whole boundary. As shown in Fig. $8(\mathrm{a})$, the solution of unknown potential, $u$, on $\frac{3 \pi}{2} \leq \theta \leq$ $2 \pi$ is more accurate when information on the known boundary is more fertile using the singular integral equation. However, for the same case the solution of unknown potential, $u$, on $\frac{3 \pi}{2} \leq \theta \leq 2 \pi$ using hypersingular 
integral equation does not have such a trend (see Fig. 8 (b)). It seems the case with information given on three quarters of the whole boundary has the best result, while the case with information given on a quarter is medium and the case with information given on half boundary is the worst. The reasons will be explained in the followings. The first reason comes from the constant element scheme we adopt. When we implement potential $u$ and the flux value $t$ both by constant elements, it results in incompatibility from these two physical quantities. As we can see from the definition of $t$, if $t$ belongs to $C^{0}$ then $u$ should belong to $C^{1}$ where $C^{m}$ means a set in which the element, should guarantee continuity of the $m$-th derivative. Therefore, if we implement $t$ and $u$ both by constant elements, theoretically speaking, it is not a physically permissible scheme since physical quantities cannot be compatible. From our experience, the hypersingular integral equation is more sensitive to such an implemented error. As we increase information on the known data, it means we have more implemented error in the known data. Thus, it results in inaccuracy in reverted information. However, as we increase information on the known data, the inverted data will become more accurate since reasonable information will reduce error. This can be understood in this way: it is well known that the laws of large number [14] tell us while more data will reduce an odd error due to a small sampling space. Therefore, as we increase elements in the hypersingular integral equation, two opposite factors will compete with each other such that we obtain our numerical result as shown in Fig. 8(b).

For the case of equal number of elements on $B_{k}$ and $B_{u}$ (cases C6, C2 and C7), 72 elements are used to discretize $B_{k}$ and $B_{u}$, and a quarter, a half and three quarters of the whole boundary are assigned as $B_{k}$. As shown in Fig. 9, the results are compared for the unknown potential, $u$, on $\frac{3 \pi}{2} \leq \theta \leq 2 \pi$. It can be found that the result for $\mathrm{C} 6$ is the best, that for $\mathrm{C} 2$ is the second and that for $\mathrm{C} 7$ is the worst. In case C6, only a quarter of the whole boundary has Cauchy data. On the other hand, a half and three quarters of the whole boundary have Cauchy data in cases $\mathrm{C} 2$ and $\mathrm{C} 7$, respectively. It seems puzzling that why increasing the boundary of known information does not increase accuracy in inverted data. Remember that in these three cases, we use equal number of elements on the known boundary and unknown boundary. While only a quarter of whole boundary has known information, the discretization error and collocation error become relatively small. As we increase the length of boundary with known information and keep equal element scheme, the disretization error and collocation error on $B_{k}$ increase such that accuracy in inverted data loses.

For the square case, the effect of distribution of known Cauchy data on the result is also examined. The Cauchy data are given on a half of the whole boundary and the distribution of boundary data has three different methods (cases S1, S2 and S3). The numerical results are illustrated in Fig. 10. It is shown that the more diversely the data distribute, the more accurately the results are obtained. This phenomenon is much more apparent in the solution of $t$.

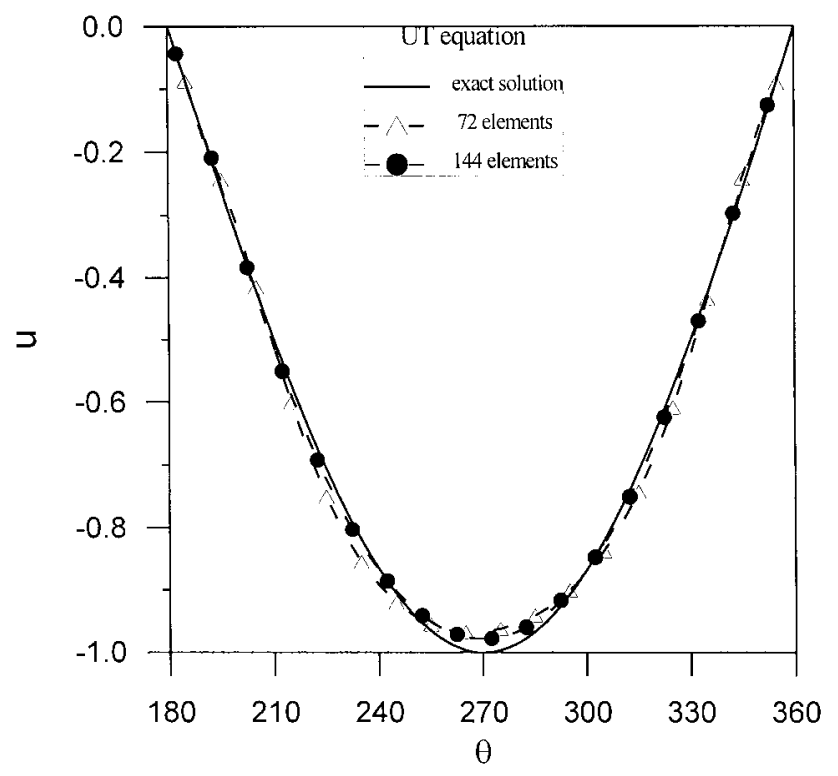

(a)

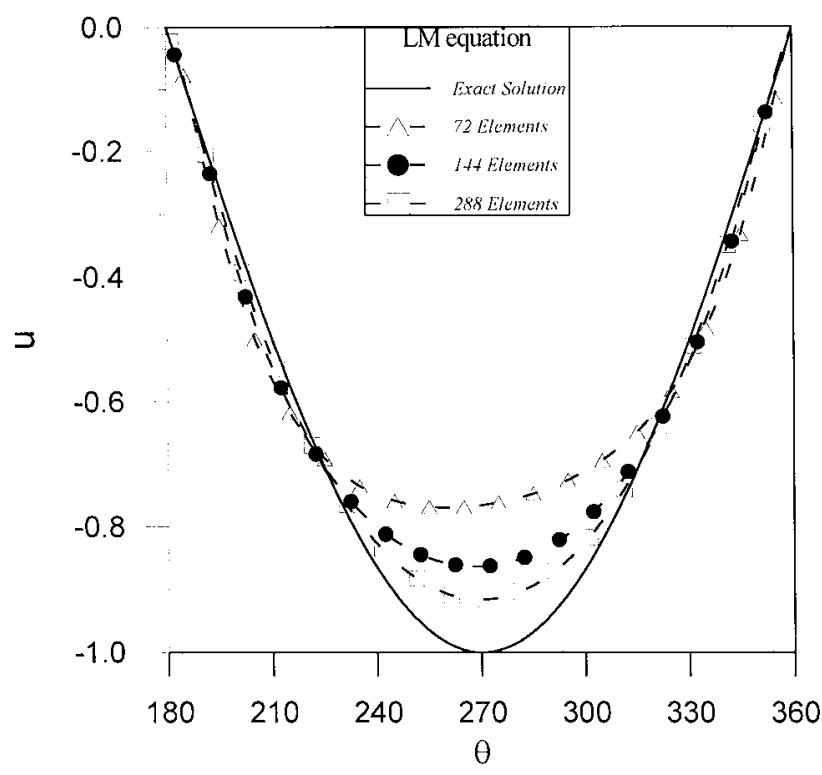

(b)

Fig. 7. (a) Convergence of solution by UT equation; (b) Convergence of solution by LM equation. 


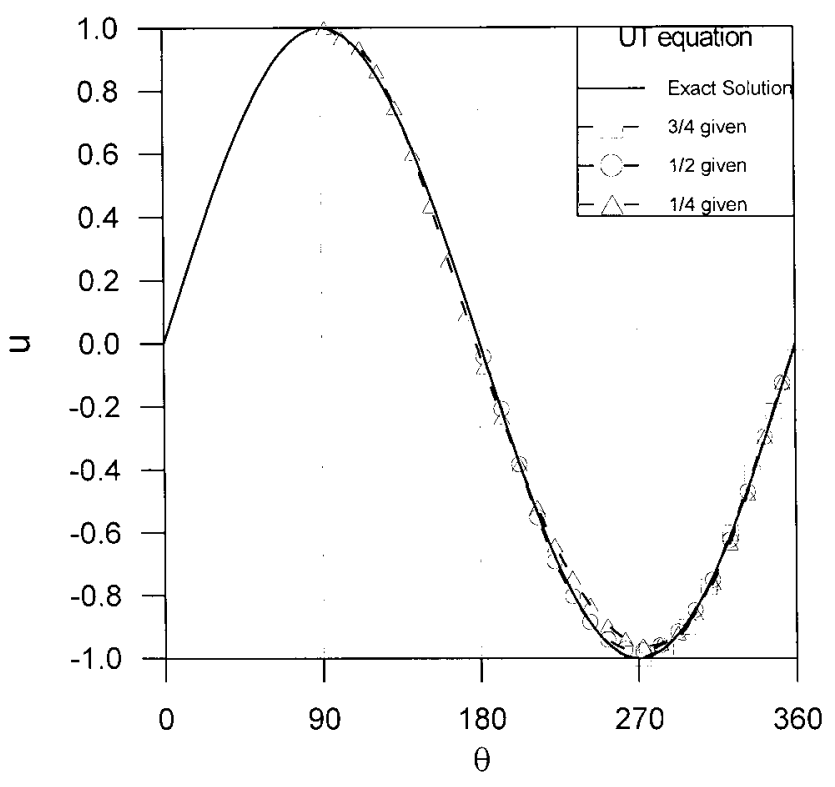

(a)

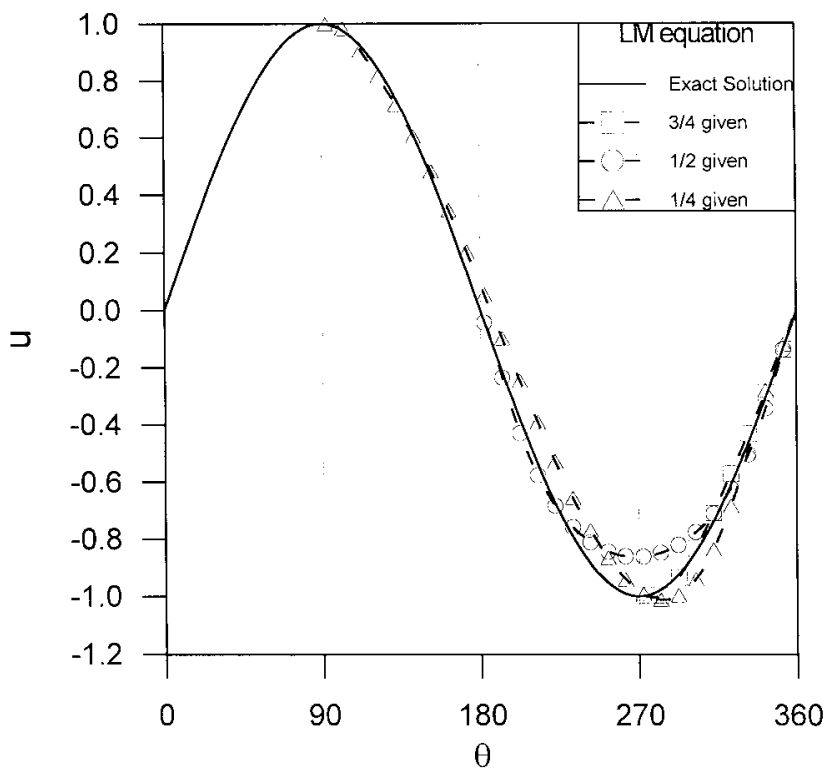

(b)

Fig. 8. (a) Effect of amount of information on the inverted data, UT method; (b) Effect of amount of information on the inverted data, LM method.

\section{CONCLUSIONS}

In this paper, the modified regularization method that combines the merits of the SVD method and Tikhonov's regularization method has been proposed to cope with the Cauchy inverse problem of the Laplace equation successfully. Owing to the ill-posed behavior

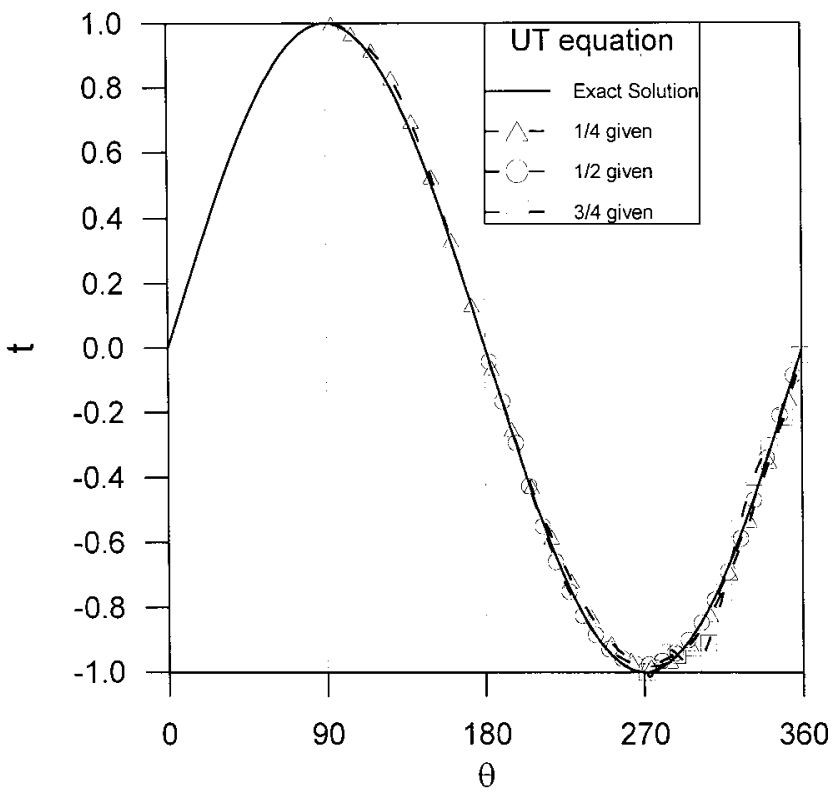

Fig. 9. Effect of data accuracy on the inverted potential, UT method.

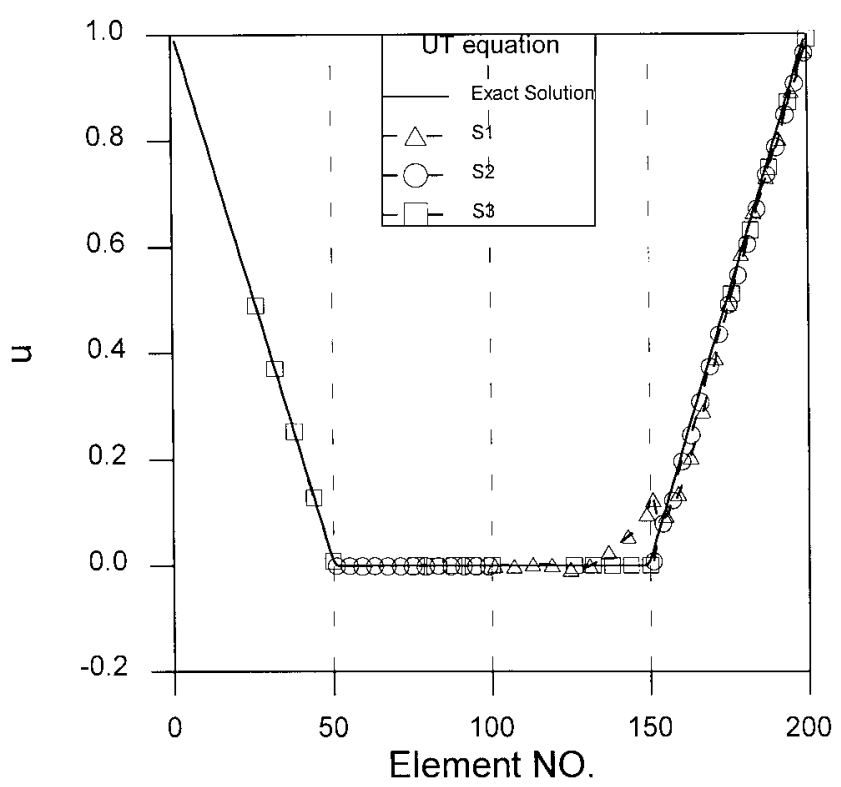

Fig. 10. Effect of diversity of known data on the inverted boundary potential, UT method.

of such an inverse problem, the L-curve concept is thus adopted to combine with the proposed approach for determining the optimal regularization parameter. Several numerical examples are also included to demonstrate how the information of data influences the solution of the problem and to show that the current approach can yield better results than the traditional two methods. It is suggested that one should obtain more 
data as diversely and accurately as possible in performing inversion process. Furthermore, the singular integral formulation is a better choice than the hypersingular integral formulation.

\section{REFERENCES}

1. Kubo, S., "Inverse Problems Related to the Mechanics and Fracture of Solid Structure," JSME International Journal, Vol. 31, pp. 157-166 (1988).

2. Hadamard, J., "Sur Les Problems anx Derivees Partielle et Leur Signification Physique," Bull University Princeton, Vol. 13 (1902).

3. Yeih, W, Koya, T. and Mura, T., "An Inverse Problem in Elasticity with Partially Overprescribed Boundary Conditions, Part I: Theoretical Approach," Transaction of ASME, J. Appl. Mech., Vol. 60, pp. 595-600 (1993).

4. Kohn, R. V. and Vogelius, M., "Determining Conductivity by Boundary Measurements II. Interior Results," Communications on Pure and Applied Mathematics, Vol. 38, pp. 643-667 (1985).

5. Yeih, W., "An Inverse Problem in Elasticity,” Ph. D. dissertation, Northwestern University, Evanston, Illinois, USA (1991).

6. Gao, Z. and Mura, T., "Inversion of Residual Stresses from Surface Displacement," Transaction of ASME, $J$. Appl. Mech., Vol. 54, No. 3, pp. 508-513 (1989).

7. Koya, T., Yeih, W. and Mura, T., "An Inverse Problem in Elasticity with Partially Overprescribed Boundary Conditions, Part II: Numerical Details," Transaction of ASME, J. Appl. Mech., Vol. 60, pp. 601-606 (1993).

8. Ivanov, V. K., "The Cauchy Problem for the Laplace Equation in an Infinite Strip," Differntsial'nye Uravneniya, Vol. 1 No. 1, pp. 131-136 (1965).

9. Tikhonov, A. N., "Solution of Incorrectly Formulated Problems and Regularization Method," Doklady Akad. Nauk SSSR, Vol. 151, No. 3, pp. 501-504 (1963).

10. Golub, G. H. and Van Loan, C. F. "Matrix Computations," Second Edition, The John Hopkins University Press, Baltimore (1989).

11. Hansen, P.C., "Analysis of Discrete Ill-posed Problem by Means of the L-curve, SIAM, Vol. 34, No. 4, pp. 561580 (1992).

12. Chen, J. T. and Hong, H.-K., "On the Dual Integral Representation of Boundary Value Problem in Laplace Equation," Boundary Elements Abstracts, Vol. 3, pp.
114-116 (1993).

13. Press, W. H., Flannery, B. P., Teukolsky, S. A., Vetterling, W. T., "Numerical Recipes: The Art of Science Computing," Cambridge University Press, Cambridge, UK (1986).

14. Roussas, G. G., "A First Course in Mathematical Statistics," Addison-Wesley Publishing Company, Reading, Massachusetts, USA (1983).

\section{以修正後之提卡諾夫正規化法求解 拉普拉斯方程之柯西問題 張 建 仁}

國立台灣海洋大學系統工程暨造船學系

葉 爲 忠謝 旻 航

國立台灣海洋大學河海工程學系

$$
\text { 摘 要 }
$$

本文針對拉普拉斯方程式中的柯西反算問題作 一研究。因著這個問題的劣性行爲, 我們使用 Tikhonov的正規化技巧並使用 L 曲線的概念來決定正 規化法當中的最佳正規化參數; 同樣的, 我們也使用 奇異值分解法加上L曲線的概念來嘗試解決這樣的問 題。數值的結果顯示當影響係數矩陣變得非常劣性化 的時候, 不論是使用Tikhonov的正規化技巧或是奇異 值分解法都並不能夠得到理想的解。因此, 本文提出 一種修正的正規化法。這種方法結合了奇異值分解法 與正規化法。由數值結果可以發現, 這種新方法優於 前兩種傳統的方法。由數值的經驗, 我們也發現對於 柯西資料反算問題, 在使用常元素時, 最好採用奇異 積分方程式會有比較準確的結果。數值結果顯示, 當 元素數目增加的時候, 收斂性變得較佳; 同時, 使用 奇異積分方程式的收斂性比較好。我們並且以數種數 值的實驗來觀察柯西資料是如何影響到反算的量。由 數值的結論, 我們認爲只要柯西資料給定的越準確, 並且在全邊界上給定的越分散, 則會有越好的結果。 